\section{MedienPädagogik}

www. medienpaed.com
Zeitschrift für

Theorie und Praxis

der Medienbildung

ISSN 1424-3636

Themenheft Nr. 15/16: Computerspiele und Videogames

in formellen und informellen Bildungskontexten

\title{
Storytelling und parasoziales Design als Motivationshilfen in Computerlernspielen
}

Matthias Bopp

\begin{abstract}
Der Artikel analysiert den möglichen Nutzen von Storytelling und parasozialem Design als Motivationshilfe in digitalen Lernspielen. Hierzu werden zunächst Zusammenhänge zwischen Storytelling, parasozialer Interaktion und Lernmotivation in Computerspielen beschrieben, darauf folgen einige Empfehlungen zur Gestaltung der Struktur von lernmotivierenden Erzählungen für digitale Lernspiele. Abschliessend werden zwei Beispiele zur Umsetzung einiger dieser Empfehlungen gegeben.
\end{abstract}

\section{Einleitung}

Computerspiele (PC Spiele, Konsolenspiele, Handheldspiele etc.) regen neben ihrem eigentlichen Zweck der Unterhaltung zu sozial erwünschten und unerwünschten Lernprozessen an und sind zugleich für viele Kinder, Jugendliche und junge Erwachsene ausserordentlich motivierend ( $\mathrm{vgl}$. den allgemeinen medienpsychologischen Überblick bei Lee \& Peng, 2006; zu kompetenzbezogenen Lernpotenzialen Gebel, 2006; zum Stellenwert in der Kinder- und Jugendkultur die KIMund JIM-Studien der letzten Jahre). Es liegt daher nahe, die Lehr-Lern-Designs dieser Spiele zu studieren, um so Anregungen zur Verbesserung der didaktischmethodischen Gestaltung von digitalen Lernspielen bzw. Bildungsspielen zu erhalten. Dabei zeigen erste Studien, dass Computerspiele (oft verdeckt) eine Vielzahl von didaktischen Methoden zu Anwendung bringen (vgl. Bopp, 2006; Gee, 2003). Der folgende Aufsatz fokussiert auf zwei dieser didaktischen Methoden, nämlich 1. auf den Einsatz von Rahmengeschichten und 2. auf die Anregung von parasozialen Interaktionen zwischen Spielern/-innen und virtuellen Charakteren zur Steigerung der Lernmotivation in digitalen Lernspielen.

Der Grund für diesen Fokus ist die grundlegende motivationale Bedeutung, die der Inszenierung von Geschichten und parasozialen Interaktionen in Computerspielen gegenwärtig zukommt (vgl. ähnlich Bateman, 2006; Gjedde, 2006; Hug 2005; luppa \& Borst, 2007; allgemein zu Storytelling und Computerspielen Atkins, 2005; Carr, 2006; Jenkins, 2003 und das Webportal http://gamesandstorytelling.net/). Dies galt bislang insbesondere für Adventure- und Single-Player-Rollenspiele, die traditionell stark storybasiert sind (vgl. z. B. die Gabriel-Knight-Serie, Silent Hill 2, The Longest Journey oder Mass Effect). Mittlerweile zeichnet sich jedoch auch 
für Genre, die traditionell nur rudimentäre Hintergrundgeschichten enthielten, ein «narrative turn» ab (vgl. z. B. Max Payne 2, Half-Life 2, Grand Theft Auto 4).

Neben diesem Wandel des Computerspiels zu einem interaktiv erzählenden Medium nehmen in Computerspielen auch parasoziale Interaktionen (PSI) und Beziehungen (PSB) an Bedeutung zu (vgl. den allgemein Literaturüberblick zu Parasozialität bei Giles, 2002). Der vieldeutige Begriff «Parasozialität» bezeichnet hier die beim Spielen auftretende Illusion einer direkten sozialen Interaktion, die damit einher gehenden Gefühle und die sich daraus ergebenden Beziehungen zwischen Spielern/-innen und den Charakteren im Spiel (vgl. Klimmt \& Vorderer, 2002).

Parasoziale Phänomene treten nicht nur im Kontext von Computerspielen auf, sondern z. B. auch, wenn Fernsehzuschauer das Gefühl haben, der Gastgeber einer TV-Show sei Teil ihrer Familie (vgl. die den Begriff einführende Studie von Horton \& Wohl, 1956; zu parasozialen Reaktionen von Kindern auf TV-Figuren vgl. Hoffner, 1996). Zu parasozialen Reaktionen zählt es auch, wenn man mit seinem Auto redet oder wenn Computernutzer ihren schlecht funktionierenden PC anschreien. Diejenigen Aspekte des Designs von Computerspielen und digitalen Lernspielen, die parasoziale Interaktionen und Beziehungen anregen sollen, werden im Kontext dieses Textes als «parasoziales Design» bezeichnet.

Im Folgenden wird zunächst ein Vorschlag dazu gemacht, wie der Zusammenhang von Lernen und Motivation sowie Storytelling und Lernmotivation in Computerspielen theoretisch modelliert werden kann. Dann werden einige Merkmale populären Storytellings in TV-Serien und Filmen aufgezeigt und ein Modell entwickelt, das es erlaubt, den Zusammenhang von Storytelling und Lernmotivation in Computerspielen und digitalen Lernspielen zu rekonstruieren und bestimmte Empfehlungen zum Storytelling in digitalen Lernspielen zu geben. Abschliessend werden diese Empfehlungen dann anhand eines konkreten Spielbeispiels veranschaulicht.

Nicht thematisiert werden in diesem Artikel die zahlreichen andere Aspekte, unter denen Storytelling in Computerspielen von Bedeutung sein kann, z. B. wenn es nicht primär als Motivationshilfe genutzt wird, sondern den Bildungsgegenstand des Spiels selbst darstellt (einen Versuch in diese Richtung bietet z. B. das interaktive Beziehungsdrama Façade) oder wenn es der Verbesserung der Verarbeitungstiefe des Lerngegenstandes dienen soll (vgl. allgemein Bruner, 2002). 


\section{Lernen, Motivation und Storytelling}

Im Folgenden wird davon ausgegangen, dass in didaktisch-methodischen Zusammenhängen Lernen als eine Form problemlösenden Handelns konzeptioniert werden kann (vgl. in allgemeindidaktischer Perspektive das Lernmodell bei Tulodziecki, Herzig \& Blömeke, 2004, 33-56, insb. 37). Lernen in Computerspielen kann dann mit Hilfe eines Prozessmodells dargestellt werden, das eine Abfolge unterschiedlicher Lernphasen beinhaltet (vgl. Abbildung 1, die eine Übertragung des Modells von Tulodziecki, Herzig und Blömeke auf das Lernen in Computerspielen darstellt).

Lernen durch problemlösendes Handeln beginnt demzufolge mit der Wahrnehmung einer gegebenen Spielsituation (1) und deren Deutung in Form einer problemzentrierten Situationsdefinition (2), z. B. in der Form: "lch stehe vor einer verschlossenen Tür, die ich öffnen muss, um im Spiel weiterzukommen.» Dieser Situationsdefinition kann nun - im lerngünstigen Fall - der Aufbau eines inneren Spannungszustands bzw. der Bereitschaft folgen, das in Frage stehende Problem handelnd zu lösen (3). Dem folgt der Abruf oder die Neukonstruktion eines passenden Handlungsziels (4), z. B. «lch muss einen schmalen Lichtstrahl erzeugen und auf die Lichtsensoren an der Tür lenken, um diese zu öffnen». Es folgt das Überdenken (5) und die Bewertung (6) verschiedener Handlungsmöglichkeiten, um dieses Ziel zu erreichen, also ein Prozess der inneren Reflexion, gefolgt von der Ausführung des ausgewählten Handlungsplans (7). Hierauf reagiert das Spielprogramm mit der Präsentation einer neuen Spielsituation (7), z. B. bleibt die Tür geschlossen und der Spieler reflektiert diese Handlungsfolge (8), beispielsweise in der Form «Die Tür öffnet sich nicht, ich habe also irgendwo einen Fehler gemacht». Dies wiederum ist bereits eine neue Situationsdefinition und damit der Beginn einer zweiten Handlungsschleife.

Begleitet sind alle diese kognitiven Prozesse vom ständigen Abruf von Wissensbeständen aus dem Arbeitsgedächtnis, von aktuellen emotionalen Zuständen, die auf kognitive Verarbeitungsprozesse Einfluss nehmen, und (bei Gruppenspielen oder Gruppenlernen) vom Gedankenaustausch mit Mitspielern. Zugleich verändern alle diese Prozesse die Inhalte des Arbeitsgedächtnisses. Soll das Spiel langfristige Wirkungen haben, müssen entsprechende Wissensbestände vom Arbeitsgedächtnis in das Langzeitgedächtnis übertragen werden. 


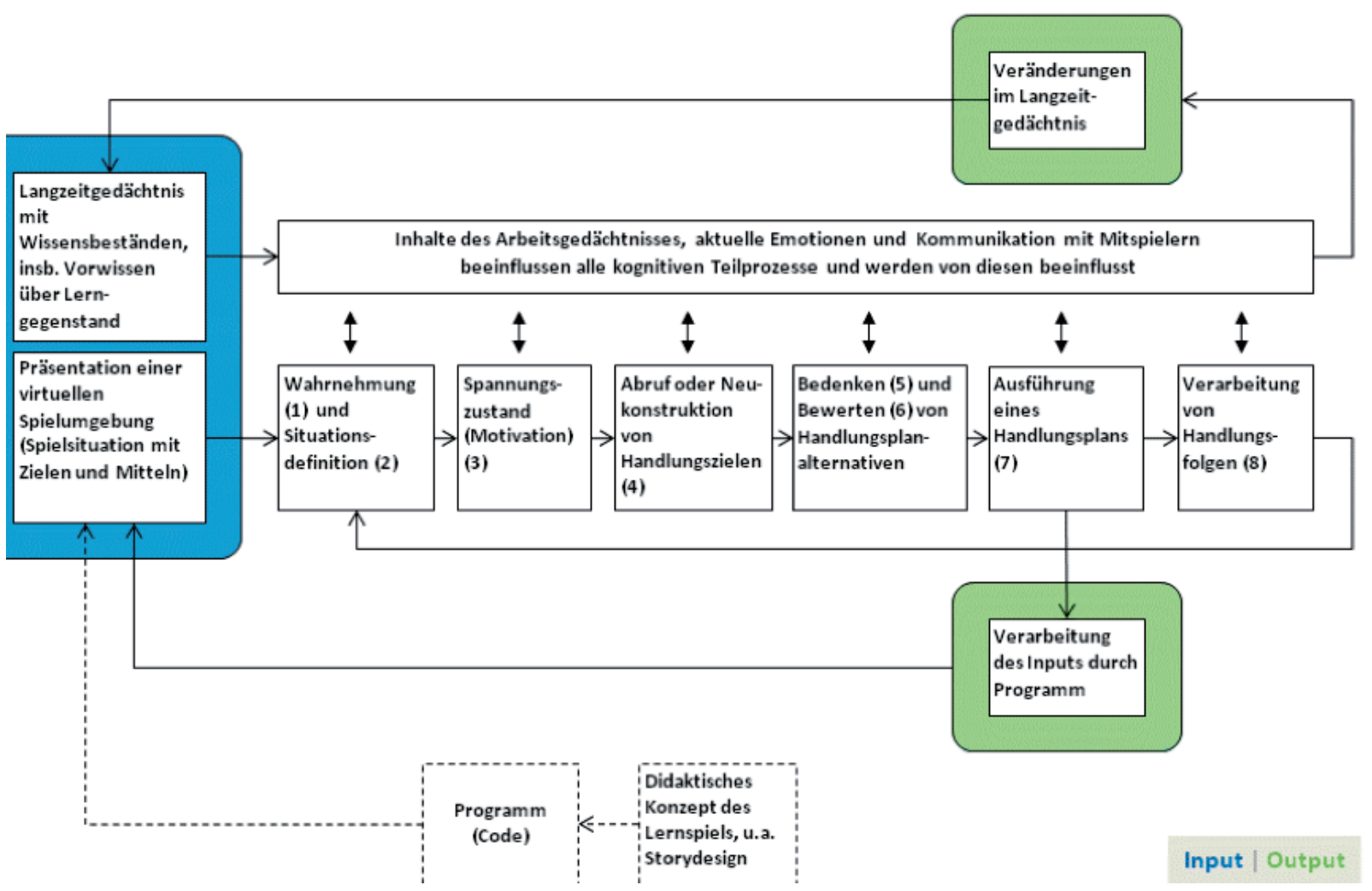

Abbildung 1. Handlungsorientiertes Lernen in Computerspielen in Anlehnung an Tulodziecki et al., 2004

Im Folgenden werden nun erweiternde Überlegungen dazu angestellt, wie digitale Lernspiele, insbesondere durch Storytelling, den Aufbau von Lernmotivation anregen können. Dabei werden zunächst einige grundlegende motivationstheoretische Überlegungen vorgetragen. «Motivation» als ein psychologischer Begriff bezieht sich üblicherweise auf die Initialisierung, Intensität und Aufrechterhaltung von Verhaltensweisen (vgl. einführend Heckhausen \& Heckhausen, 2006). Um Motivationsprozesse in Computerspielen umfassend beschreiben und erklären zu können, müssen vielfältige Variablen berücksichtigt werden. Diese lassen sich drei Gruppen zuordnen. Die erste Gruppe beinhaltet relativ stabile Merkmale, die ein Spieler mitbringt, wenn er in eine Spielsituation eintritt. Hierzu zählen das im Langzeitgedächtnis verankerte bereichsspezifische Wissen und Können, Alter und Geschlecht u. a. Diese Merkmale können ihrerseits als das Resultat einer bestimmten Lernbiographie in bestimmten sozialen Kontexten und auf der Grundlage von bestimmten Anlagen rekonstruiert werden. Die zweite Variablengruppe bilden die zahlreichen innerpsychischen Prozesse während des Spielens, also bestimmte kognitive, emotionale und physiologische Prozesse (wie etwa Flow-Zuständen). Eine dritte Merkmalsgruppe umfasst die äussere Situation des Spielers und hier vor allem die virtuelle Spielumgebung. Eine alle diese Variablengruppen integrieren- 
de Motivationstheorie des Computerspiels liegt derzeit nicht vor (vgl. verschiedene Ansätze bei Vorderer \& Bryant, 2006, 91-194; für die deutschsprachige Diskussion Fritz, 1995).

Das Storytelling eines Computerlernspiels gehört offenbar zu dieser letzten Gruppe. Im Folgenden wird es in seinem Zusammenhang mit bestimmten kognitiven und emotionalen (also inneren) Prozessen und Merkmalen eines Spielers beschrieben. Bei diesen inneren Prozessen handelt es sich um Erwartungen und Bewertungen, die mit Lernhandlungen verknüpft sind.

\section{Ansatzpunkte für Storytelling als Motivationsmittel}

Rheinberg (2005) hat ein Modell vorgelegt, das deutlich macht, welchen Einfluss bestimmte Erwartungen und Bewertungen auf die Handlungsmotivation im Allgemeinen haben (vgl. zu den kognitionspsychologischen Grundannahmen dieses Modells Anderson, Funke \& Plata, 2007; zum Zusammenhang von Kognitionen, Gefühlen und Körperzuständen bei Lernprozessen im Computerspiel Anderson \& Buckley, 2006). Er betrachtet Handlungsmotivationen als das Ergebnis des Zusammenspiels von (mehr oder minder bewussten) Prozessen der Definition einer Ausgangssituation, der Konstruktion von Erwartungen über die Folgen von Handlung oder Nicht-Handeln in dieser Situation und der emotional gefärbten Bewertungen. Angewendet auf die Motivation eines Schülers für eine bestimmte Klausur zu lernen, ergibt sich dann beispielsweise folgendes Prozessschema (vgl. Abbildung 2):

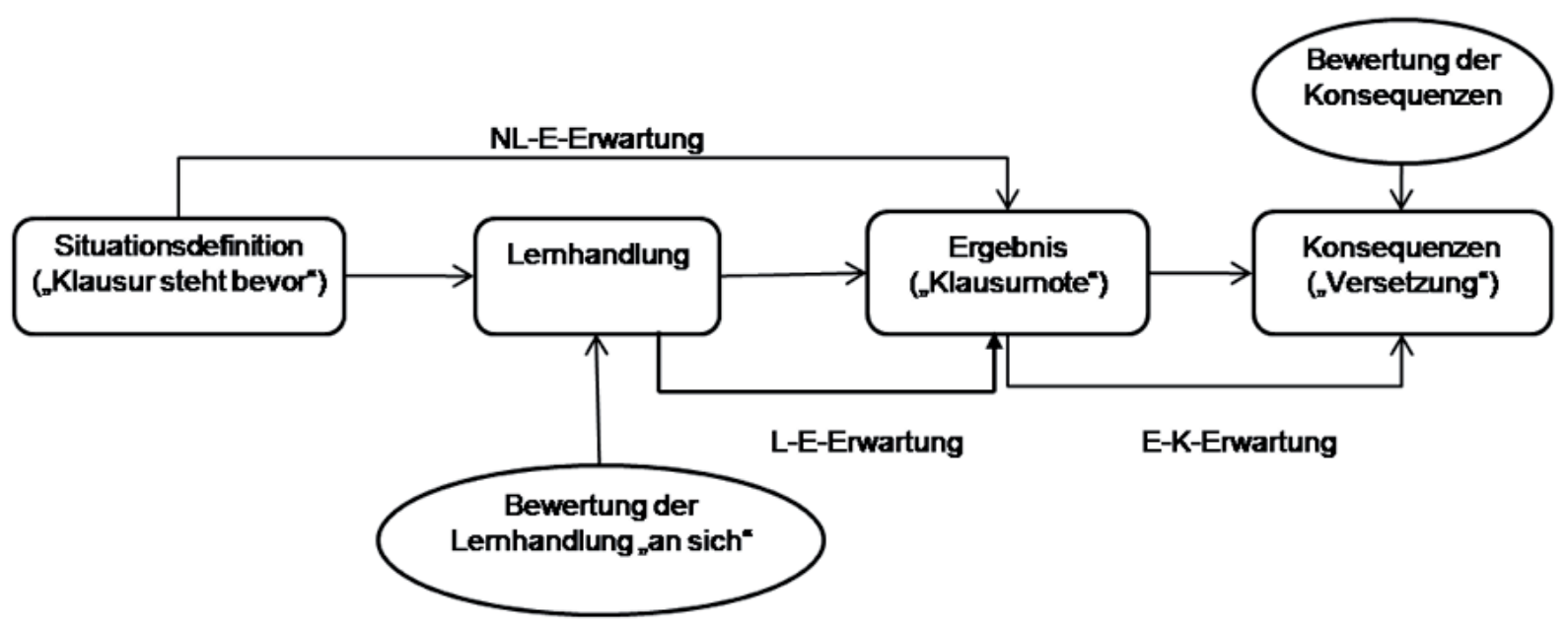

Abbildung 2. Kognitives Lernmotivationsmodell, angelehnt an Rheinberg (2005) 
Zunächst nimmt ein Schüler eine bestimmte Situationsdefinition vor, z. B. «In einer Woche findet eine Physikklausur statt». Hieran anknüpfend kommt es zur Bildung von drei Typen von Erwartungen. Der erste Typ bezieht sich darauf, was das Ergebnis («E») des Nicht-Lernens («NL») in der Situation sein wird («NL-E-Erwartung»). Je nach Selbstbild und Erfahrungen mit zurückliegenden Klausuren etc. (also Merkmalen, die er beim Eintritt in die Situation mitbringt, siehe oben die erste Gruppe der Motivationsvariablen) kann eine solche NL-E-Erwartung z. B. sein: «lch bestehe die Klausur auch ohne dafür zu lernen» oder «Wenn ich nichts tue, falle ich durch».

Der zweite Typ von Erwartungen betrifft das, was geschehen wird, wenn sich der Schüler auf die Klausur vorbereitet, also Lernhandlungen vollzieht («L» für Lernhandlungen). Eine solche denkbare «L-E-Erwartung» wäre z. B. «lch falle auf jeden Fall durch, Physik verstehe ich einfach nicht» oder aber «Wenn ich mit Steffi zusammen lerne, bekomme ich bestimmt eine 2».

Der dritte Erwartungstyp betrifft die mittelbaren oder langfristigen Konsequenzen ( KK»), die das Ergebnis («E») einer Lernhandlung (hier also eine bestimmte Klausurnote) haben wird, also beispielweise soziale Anerkennung durch Eltern oder eine Nichtversetzung («E-K-Erwartungen»).

Neben diesen Erwartungen haben zwei emotional gefärbte Bewertungsprozesse einen wesentlichen Einfluss auf die Handlungsmotivation. Zum einen ist das die Festlegung des Wertes der möglichen Handlung an sich (z. B. «Physik-Lernen macht mir Spass, egal was das für Auswirkungen auf meine Note hat»). Zum anderen ist dies die Bewertung der mittelbaren oder langfristigen Konsequenzen eines bestimmten Handlungsergebnisses, z. B. «Was soll's, wenn ich wegen Physik den Hauptschulabschluss nicht schaffe, ich kann nach der Schule ja auf jeden Fall in der Werkstatt von Onkel Walter arbeiten».

Rheinbergs Modell verdeutlicht, welche emotionalen und kognitiven Prozesse zu hohen und insbesondere auch zu niedrigen Graden an Lernmotivation führen können.

Bei sonst gleichen Bedingungen werden z. B. solche Schüler eine geringere Lernmotivation haben,

1. die Lernhandlungen an sich keinen Eigenwert zumessen (niedrige «intrinsische» Motivation)

2. die ihre eigene bereichsspezifische Lernfähigkeit unter- oder ihr Wissen überschätzen, also über unrealistische NL-E- oder L-E-Erwartungen verfügen

3. die nicht external motiviert sind, weil ihnen die negativen Konsequenzen ihres Tuns nicht klar sind (sie erkennen z.B. nicht, welche Folgen es haben kann, ohne Abschluss die Schule zu verlassen)

4. die nicht external motiviert sind, weil sie sich zwar über die Konsequenzen ihres Handelns im Klaren sind, diese Konsequenzen aber letztlich falsch, z. B. verharmlosend bewerten. 
Dieses Motivationsmodell vorausgesetzt, ergeben sich für das Storytelling von Lernspielen verschiedene Ansatzpunkte, von denen hier drei besonders hervorzuheben sind.

1. Es kann bei Lernern mit einer geringen intrinsischen Motivation versucht werden, negative Voreinstellungen und damit Bewertungen von Lernen an sich zu verändern. Beispielsweise kann die Geschichte von NPCs (non-player characters - vom Computer gesteuerte Spielcharaktere) und Avataren (vom Spieler gesteuerte Charaktere) präsentiert werden, die dem Lernen gegenüber positive Werthaltungen zeigen und deren Einstellungen den Spieler über Identifikationsprozesse beeinflussen können (vgl. hierzu Gee, 2003, der dies am Beispiel von Lara Croft illustriert, die aus Gees Sicht eine bestimmte Forschungsmentalität personifiziert).

2. Es kann versucht werden, durch eine Rahmenerzählung bei Lernern mit einem zu geringen Vertrauen in die eigene Lernfähigkeit deren Selbstwirksamkeitseinschätzungen und daraus folgende L-E-Erwartungen zu korrigieren (vgl. das Konzept der self-efficacy bei Bandura, 1977). Ansatzpunkte hierfür sind bestimmte Identifikations- bzw. Rollenangebote in einer storybasierten Spielwelt, entsprechende sprachliche Aufmunterungen durch NPCs u.a.

3. Es besteht die Möglichkeit, über eine bestimmte Rahmengeschichte Lernhandlungen mit Konsequenzen in der Spielwelt zu verbinden, die für den Lerner wünschenswert erscheinen. Ein Beispiel hierfür ist eine Spielsituation, in der der Spieler etwas lernen muss, um einem Spielcharakter zu helfen, der ihm «am Herzen liegt», oder einem Charakter zu schaden, dem er gegenüber feindselig eingestellt ist (zu solchen parasozialen Interaktionen siehe unten).

Dies sind im Prinzip weder in der didaktischen Theorie oder in psychologischen Lerntheorien, noch in der pädagogischen Praxis unbekannte Motivationshilfen. Auf lernpsychologischer Ebene stehen sie z. B. konstruktivistischen Lehr-Lern-Theorien nahe (anchored instruction, situiertes Lernen, cognitive apprenticeship; vgl. Terhart 2005). Auf praktischer Ebene finden sich Rahmenerzählungen im hier vorgeschlagenen Sinne z. B. in den Lehrfilmen der bekannten Adventures of Jasper Woodbury-Reihe (vgl. Cognition and Technology Group at Vanderbilt, 1997). Dort wird Jasper, der junge Held, mit verschiedenen praktischen Problemen konfrontiert, die er nur lösen kann, wenn er bestimmte mathematische Fähigkeiten anwendet. So muss er z. B. Dreieckswinkel errechnen, um dann mit einem Gleiter von einem Berg aus einen verletzen Adler möglichst schnell zu einem Veterinär bringen zu können. Bestimmte Varianten des Rollen- und Planspieles arbeiten ebenfalls mit dem Konzept der Identifikation mit lern- und bildungsfreudigen Charakteren (vgl. für den Bereich der politischen und wirtschaftlichen Bildung Klippert, 2008).

Will man diese traditionellen Methoden im Rahmen von Computerlernspielen einsetzen, dann müssen sie jedoch diesem neuen Medium und seinen besonderen 
Möglichkeiten und Restriktionen angepasst werden. Hierzu ist es u. a. sinnvoll zu klären, was Kennzeichen erfolgreicher Formen des Storytelling in Computerspielen sind, denn ohne eine für den Lerner interessante bzw. spannende Geschichte mit entsprechenden Charakteren werden die oben angesprochenen Anregungen zur Veränderung von lernbezogenen Erwartungen und Bewertungen wenig Wirkung haben. Im Folgenden wird deshalb der Frage nachgegangen, welche Empfehlungen sich zur Gestaltung einer interessanten bzw. immersiven Erzählung vor dem Hintergrund des Forschungstandes abzeichnen.

\section{Ein Strukturmodell populären Storytellings in Computerspielen}

Zur Beantwortung der Frage, welche Kennzeichen eine Geschichte zu einer interessanten Geschichte für ein breites Publikum machen, kann man sich in pragmatischer Hinsicht zunächst an creative writing guidebooks orientieren (vgl. McKee, 1997; Field, 2005). Entsprechende Ratgeber berufen sich üblicherweise auf die Analyse populärer Spielfilme und TV-Serien und kommen dabei zu dem Schluss, dass eine interessante Handlung im Kern aus einer Serie von Ereignissen um einen (oder mehrere) Hauptcharakter (Held, Protagonist) herum besteht. Dieser Held hat ein Hauptziel und steht bei der Erreichung dieses Ziels einer Vielzahl von Hindernissen gegenüber. Typischerweise sind dies andere Personen (die "Schurken»), die Natur (Moby Dick, Dr. House) oder auch innere Konflikte des Helden selbst (Hamlet). Zumindest der Hauptcharakter sollte dabei multidimensional sein, also Stärken, Schwächen und Motive haben, die erkennbar in seiner Biographie wurzeln und sein Handeln beeinflussen.

In Geschichten für Kinder und Jugendliche sind solche Hauptziele häufig bildungsbezogene Entwicklungsaufgaben wie das Erwachsenwerden und seinen Platz in der Welt finden (vgl. Rojany-Buccieri \& Economy, 2005; Lamb, 2001; zu einer Übersicht zu typischen Entwicklungsaufgaben von Kindern und Jugendlichen vgl. Oerter \& Montada, 2008).

Empirisch fundiert und differenziert werden können solche Empfehlungen zur Struktur von Unterhaltungsgeschichten durch Zillmanns medienpsychologische Untersuchungen zu kognitiven und emotionalen Reaktionen von Zuschauern auf erfolgreiche spannungsorientierte Filme und TV-Soaps (vgl. Zillmann, 1996, 219; Abbildung 3). 


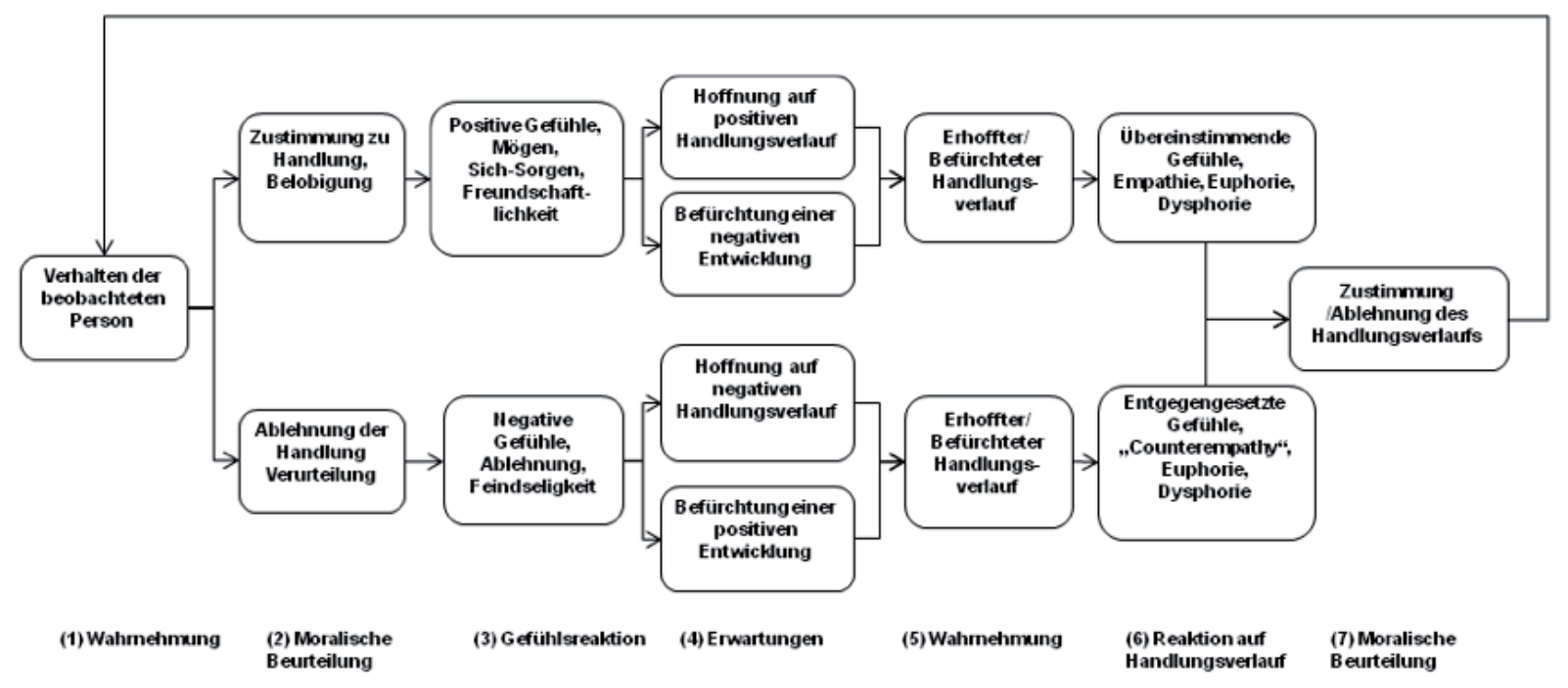

Abbildung 3. Rezeptionsmodell von unterhaltungsorientierten Filmen und TV-Soaps, angelehnt an Zillmann (1996)

Zillmann zufolge können die Reaktionen von Zuschauern auf das Geschehen in Form von Rezeptionseinheiten dargestellt werden. Zu Beginn einer solchen Episode bewerten Zuschauer die Handlungen einzelner Charaktere auf Grund ihrer moralischen Überzeugungen und ordnen sie einer von zwei Figurengruppen zu: der Gruppe der positiv besetzten Charaktere (der/die Protagonisten und diesen freundlich gesonnene Nebenfiguren) oder der Gruppe der «Schurken». Zwischen beiden Gruppen bestehen grundlegende Konflikte, die zu Bedrohungen für die Protagonisten und der Erreichung ihrer Ziele führen. Bei den positiven Charakteren finden sich oft Liebespaare mit mehr oder minder ausgeprägten Beziehungskonflikten. In manchen Erzählungen sind einzelne Charaktere nicht leicht einer dieser beiden Gruppen zuzuordnen (z. B. in der Serie Lost).

Die Einordnung von Handlungsfiguren zu einer dieser Gruppen führt dann weiter zur Entstehung von emotionalen Beziehungen zwischen Rezipient und Figuren. Bezüglich der positiven Figuren sind dies Gefühle der Sympathie, der Fürsorge und eventuell der Identifikation, bei negativen Charakteren sind es Antipathie und Feindseligkeit. Diese Gefühle wiederum führen zu bestimmten Erwartungen - genauer zu Hoffnungen und Befürchtungen - hinsichtlich des weiteren Handlungsverlaufes: Bezüglich der positiven Charaktere erhofft man, dass sie ihre Ziele erreichen und man befürchtet, dass ihnen Unheil widerfährt, bezüglich negativer Figuren befürchtet man umgekehrt, dass sie ihre Ziele erreichen können, und hofft auf deren Scheitern.

In Reaktion auf den folgenden Handlungsverlauf entwickeln Zuschauer dann in einer letzten Phase bezüglich positiver Charaktere Gefühle der Freunde oder 
des Mitleids (je nachdem, was mit ihnen passiert) und hinsichtlich der Schurken wiederum spiegelverkehrt Gefühle der Schadenfreude oder des Triumphes, je nachdem, ob deren Pläne erfolgreich sind oder nicht. Es folgt eine erneute moralische Beurteilung, die wiederum den Beginn einer neuen Rezeptionsepisode bilden kann usw.

Je nach Erzählformat können mit solchen Erzähl- und Rezeptionseinheiten unterschiedliche und sich historisch wandelnde Erzählmuster gebildet werden. In einem Kinofilm etwa müssen andere Spannungsbogen aufgebaut werden als in einer TVSerie. Ein weit verbreitetes Erzählmuster für eine bestimmte Form von TV-Soap beinhaltet z. B., dass es zu jeder Zeit drei parallele Handlungsstränge gibt, die in sich jeweils aus einer Exposition, einem Mittellteil und einer Auflösung bestehen. Darüber hinaus finden sich in jeder einzelnen Episode der betreffenden Soap die Exposition eines neuen Handlungsstrangs, der Mittelteil eines Strangs, der in der letzten Episode begonnen wurde, sowie die Auflösung eines dritten Strangs, der in der vorletzten Episode begann.

Zillmanns Modell ist anhand nicht-interaktiver Erzählformen entwickelt worden, lässt sich aber modifiziert auf Erzählstrukturen und deren Rezeption in Computerspielen anwenden. Modifikationen des Modells müssen hier vor allem dem interaktiven Charakter dieser Erzählform gerecht werden (vgl. Abbildung 4). Dieser hat einerseits Einfluss auf die Erzählstruktur selbst und andererseits auf die Reaktionen des Spielers auf diese Strukturen.

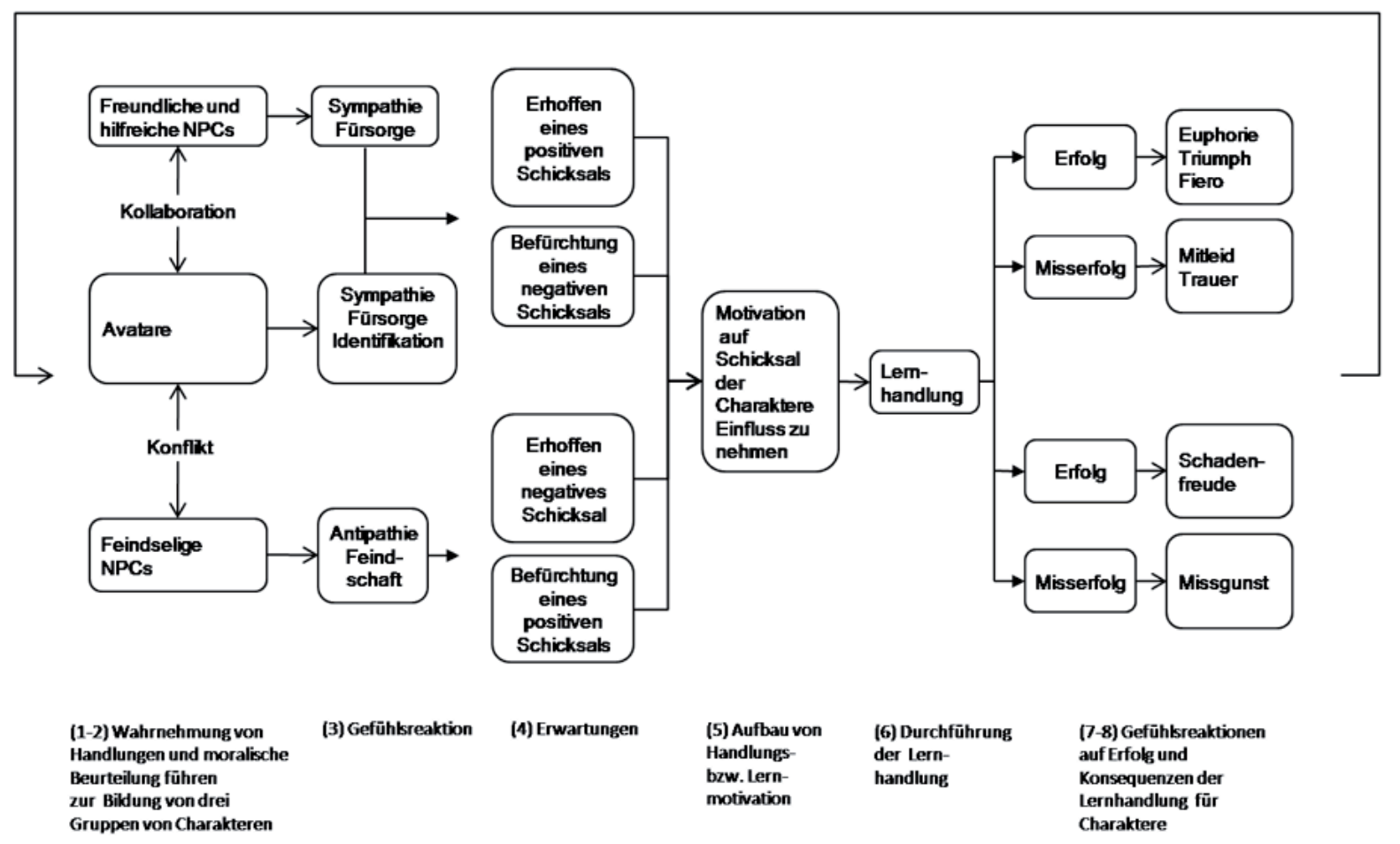

Abbildung 4. Rezeptionsmodell interaktiver Erzählungen in Computerspielen 
Zunächst zur Erzählstruktur. Erstens kann der Spieler beim Computerspiel im Gegensatz zu traditionellen Erzählmedien über die Handlungen seines Avatars Einfluss auf den Handlungsverlauf nehmen (und sei es auch nur, dass sich dieser in einer linearen, vom Programm vorgegebenen Weise entwickelt). Dementsprechend ist es sinnvoll hier nicht zwei, sondern drei Gruppen von Charakteren zu unterscheiden: freundliche und hilfreiche NPCs, feindselig gestimmte NPCs und dazwischen den oder die Avatare. Beispiele für den Versuch, eine Gruppe von positiv besetzten Charakteren zu schaffen, die den Avatar unterstützt und von ihm unterstützt wird, finden sich in Half-Life 2 und den Episoden 1 und 2 dieser Reihe (siehe Abbildung 5; vgl. zu typischen emotionalen Reaktionen in Computerspielen auch die aufwendige qualitative Rezeptionsstudie von Lazarro, 2004).

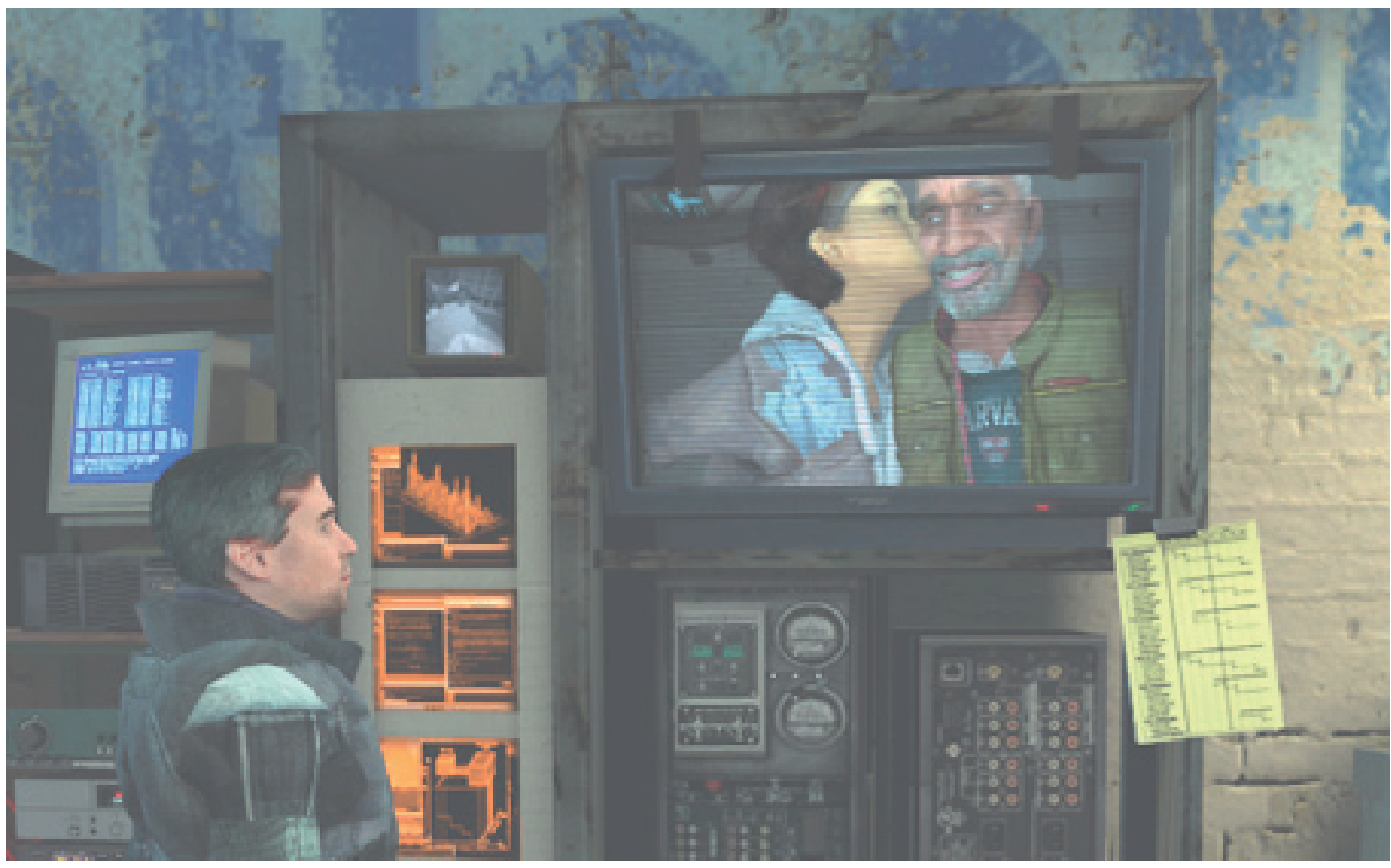

Abbildung 5. Screenshot aus Half-Life 2

Zweitens scheint die Länge eines Computerspiels (die von ca. 10 Stunden bei Shootern bis zu mehr als 100 Stunden bei bestimmten Rollenspielen reichen kann) das Format des episodischen Erzählens nahe zu legen, das auch in TV-Serien verwendet wird. Ein innovatives Beispiel für diese Erzählform ist aktuell Alone in the Dark 5, das seinen Handlungsverlauf explizit in etwa 45-minütige Einheiten aufteilt und Zusammenfassungen des bisher Geschehenen enthält. 
Drittens bedingt der interaktive Charakter von Erzählungen in Computerspielen, dass sich gegenüber traditionellem Erzählen die Art der Motivation im Umgang mit der Erzählung verändert. In nicht-interaktiven Erzählformen wird primär die Motivation erzeugt, der Handlung weiter zu folgen. Man verlässt nicht das Kino solange der Film läuft und man schaltet den Fernseher nächste Woche wieder ein. In Computerspielen hingegen bildet sich die Motivation, handelnd auf den Verlauf der Geschichte und die Schicksale der Charaktere einzuwirken. Hierzu passend muss dann die Struktur der Geschichte entsprechende Eingriffsmöglichkeiten enthalten. In Lernspielen sind diese Eingriffsmöglichkeiten demgemäss an zuvor zu vollziehende Lernhandlungen gebunden (vgl. Abbildung 5).

Hinsichtlich der kognitiven und emotionalen Reaktion von Spielern ergibt sich aus dem interaktiven Charakter des Storytellings in Computerspielen erstens, dass als Reaktionstyp verstärkt die einleitend angesprochenen Formen parasozialer Interaktionen auftreten. Diese sind in Ansätzen auch bei der Rezeption von TV-Sendungen zu beobachten, etwa wenn Fussballfans «Schiess doch» schreien, wenn ein Stürmer im Ballbesitz dem gegnerischen Tor gegenüber steht. Auch kleine Kinder scheinen, wenn sie noch nicht deutlich zwischen Medieninhalten und der aussermedialen Realität unterscheiden können, generell zu solchen Reaktionen zu neigen. In Computerspielen treten parasoziale Reaktionen aber vermutlich verstärkt und auch in anderen Altersgruppen auf. Die empirische mediendidaktische Forschung zu parasozialen Effekten steht gegenwärtig noch am Anfang. Zu analytischen Zwecken scheint es sinnvoll, hier in der Zukunft drei Forschungsfelder zu unterscheiden.

- Das erste Feld umfasst Auslöser parasozialer Reaktionen innerhalb der virtuellen Spielwelt. Zu solchen Auslösern zählt generell alles, was die Illusion einer direkten sozialen Interaktion fördert. Hierzu zählen vermutlich insbesondere bestimmte Merkmale von NPCs und Avataren. Wenn deren Äusseres z. B. realistisch wirkt (was es in zunehmendem Masse tut, vgl. Abbildung 6), wenn sie soziale Rollen überzeugend ausfüllen, Mimik zeigen, wenn sie den Spieler ansprechen und mit ihm und untereinander Augenkontakt halten, dann erhöht dies die Wahrscheinlichkeit parasozialer Reaktionen (vgl. Nass \& Steuer, 1993). Darüber hinaus liegt die Vermutung nahe, dass die Einpassung von Charakteren in eine interessante Erzählhandlung parasoziale Reaktionen befördert, wenn dies den Figuren mehr Tiefe verleiht.

- Das zweite Feld umfasst die Untersuchung der verschiedenen Reaktionen (Gedanken, Gefühle und insbesondere Handlungen) von bestimmten Spielern auf dieses parasoziale Design. Beispiele hierfür sind moralische Beurteilungen von Figuren, der Aufbau einer emotionalen Beziehung zu diesen, der Wunsch, sich für bestimmte NPCs einzusetzen oder diesen zu schaden, das tatsächliche Handeln auf Grund solcher Emotionen etc. 
- Ein drittes Untersuchungsfeld beinhaltet Persönlichkeitseigenschaften von Spielern, die Einfluss auf die Art der Reaktionen haben (offenbar reagiert nicht jeder Spieler auf NPCs mit den gleichen parasozialen Reaktionen oder überhaupt auf parasoziale Weise).
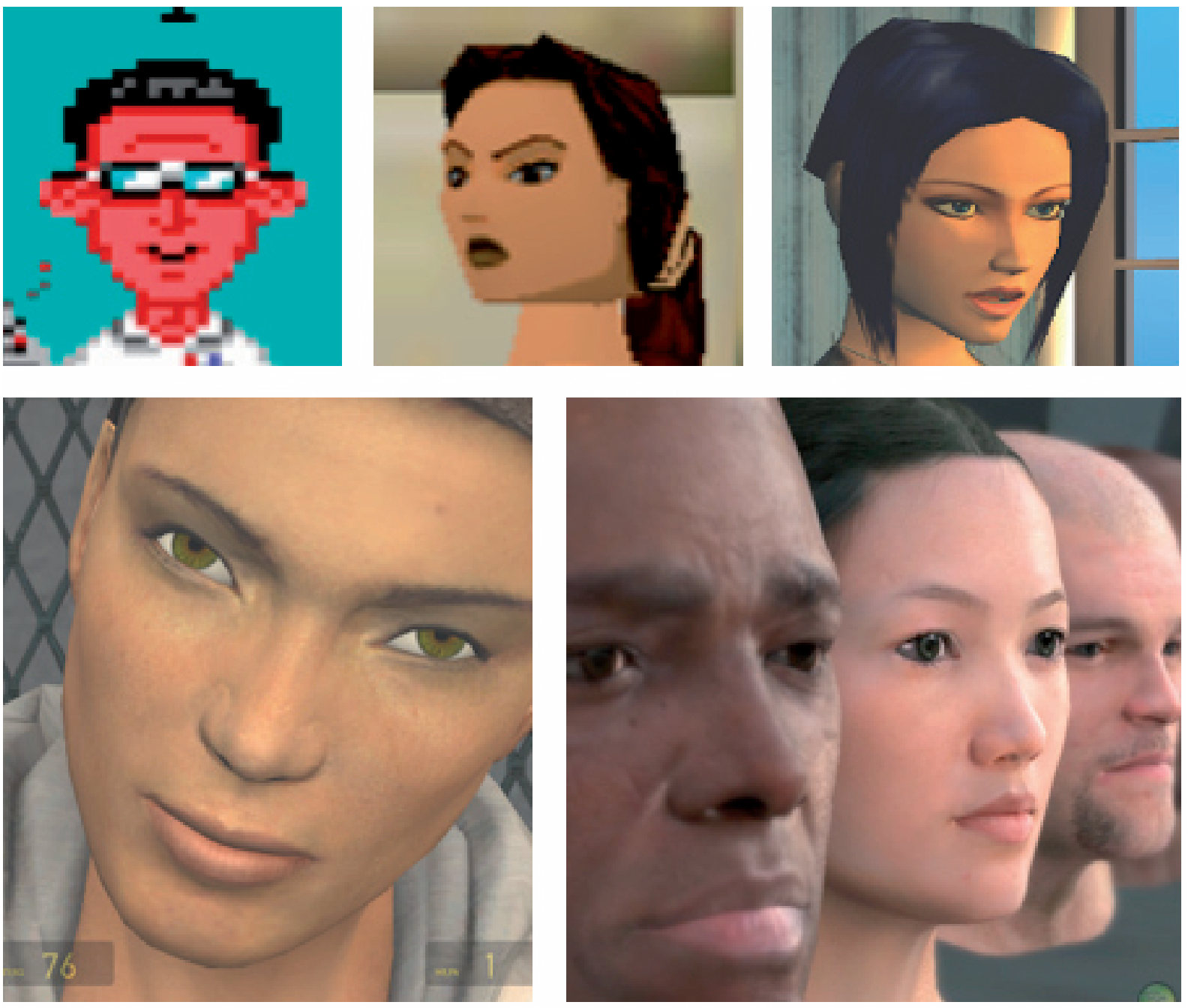

Abbildung 6. Zunahme des grafischen Realismus von Computerspielfiguren in den letzten 20 Jahren: Maniac Manson (1987), Tomb Raider II (1997), Broken Sword III - The Sleeping Dragon (2003), Half-Life 2 (2004), Pressebild zu Crysis (2007) 
Neben parasozialen Interaktionen betrifft eine zweite Auswirkung des interaktiven Charakters von Computerspielen vermutlich eine verstärkte Tendenz, mit der Handlung und den Charakteren zu spielen, also das zu betreiben, was gelegentlich «Play the Game» genannt wird. Natürlich kann man auch Filme etc. "gegen den Strich» rezipieren, das Computerspiel legt dieses Verhalten aufgrund seines Spielcharakters aber traditionell besonders nahe. Dieser Hinweis macht im Übrigen deutlich, dass die hier aufgezeigten Strukturen interaktiver Erzählungen die tatsächlichen Reaktionen von Spielern nicht determinieren können. Hinsichtlich der Wirkungen von Storytelling auf Rezeption und Lernmotivation sind nur probabilistische Aussagen möglich.

\section{Zwei Anwendungsbeispiele}

Die praktische Anwendung des hier vorgestellten Storytellingkonzepts wird im Folgenden am Beispiel der Lerndemo ELEKTRA und dem Politikspiel Global Conflicts: Palestine veranschaulicht. ELEKTRA entstand im Rahmen eines von der Kommission der Europäischen Union finanzierten interdisziplinären Forschungsprojekts (Enhanced Learning Experience and Knowledge Transfer, FP6-IST-027986), an dem auch der Autor beteiligt war. Hauptziele des Projekts waren die Entwicklung einer Methodik zu Entwurf und Produktion innovativer digitaler Lernspiele und die Entwicklung einer Lernspieldemo, die entsprechende methodische Empfehlungen umsetzt (nähere Informationen und Publikationen unter http://www.elektra-project.org).

Die so entstandene ELEKTRA-Lerndemo ist ein Physikspiel für die Mittelstufe allgemeinbildender Schulen. Das Spiel beginnt mit einem kurzen Introfilm, der in die Hintergrundgeschichte einführt. Setting und Plot folgen den Mustern eines Mystery Adventures, ähnlich z. B. entsprechenden populären Romanen von Dan Brown und der Baphomet-Adventure-Spiel-Reihe. Galileo Gallei hat ein geheimes Tagebuch hinterlassen, welches vermeintlich unvorstellbare Macht verleiht und dem zwei verfeindete Gruppen auf der Spur sind. George (der Avatar des Spiels) und die junge Lisa werden versehendlich in den Kampf der beiden Gruppen hineingezogen. Unterstützt werden sie dabei vom «Geist» Galileos. 


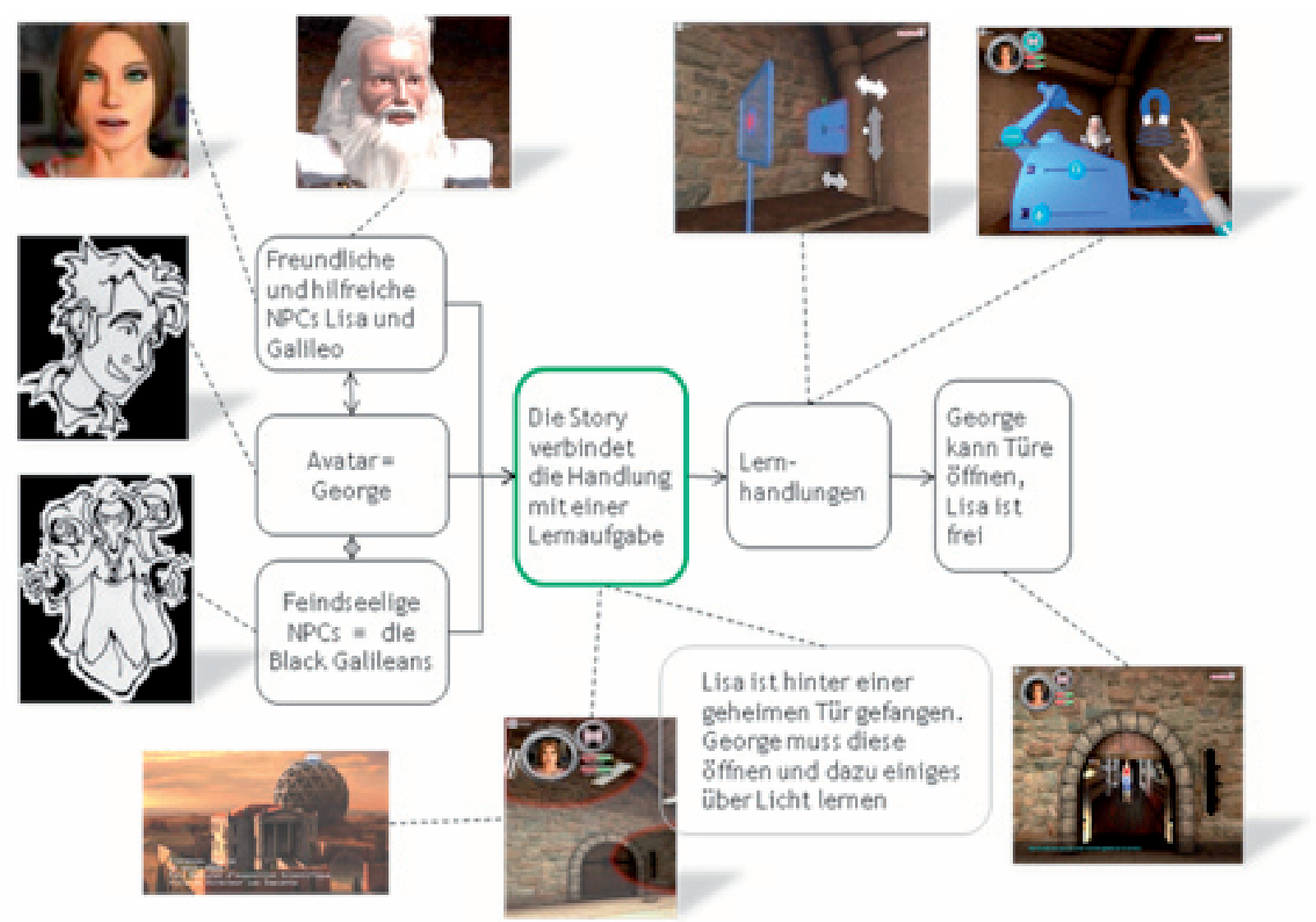

Abbildung 7. Storystruktur der Physiklerndemo ELEKTRA

Die Wahl einer Geschichte mit Bezug zur Renaissance und Galileo Galilei ermöglicht dabei zahlreiche Bezüge zu den Anfängen der modernen Naturwissenschaft. In das Zentrum der Handlung einen jungen Mann und eine junge Frau zu stellen, bietet zum einen Identifikationsmöglichkeiten für die Spieler/innen, zum anderen erlaubt es, romantische Aspekte in die Handlung einzubeziehen, ein Thema, das für Jugendliche von besonderem Interesse ist.

Die Verknüpfung von Lernhandlungen mit der Geschichte geschieht im Spiel auf folgende Weise: Im Laufe der Handlung wird Lisa in einem Geheimlabor hinter einer verborgenen Tür eingesperrt. George muss sie befreien, indem er die Tür öffnet. Hierzu ist es nötig, einen Lichtstahl in einem bestimmten Winkel auf den Öffnungsmechanismus der Tür zu richten, und dafür muss George zunächst das physikalische Konzept des Lichtstrahls und wie man es zweidimensional abbilden kann, verstanden haben (ein in der EU üblicher Lernstoff für die 7. oder 8. Jahrgangsstufen). Um sich dieses Wissen anzueignen, kann er im Labor mit Galileos Hilfe verschiedene Experimente machen (vgl. Abbildung 7). 
Eine intern durchgeführte Evaluation in einigen französischen Mittelstufenklassen zeigte zwar, dass Setting, Geschichte und Charaktere des Spiels als ansprechend und interessant empfunden wurden (vgl. Linek u. a., 2008). Dennoch wirkt die Einbindung der eigentlichen Lernhandlungen in den Spielverlauf etwas erzwungen, was auf ein besonderes kreatives Problem bei der Entwicklung von storybasierten Lernspielen hinweist: Wie ist eine wirklich überzeugende Verbindung von interessanter Geschichte und bildungsrelevanten Lerninhalten möglich?

In zahlreichen älteren digitalen Lernspielen ist dieses Problem nur unzureichend gelöst und gefährdet damit die Immersion in die Geschichte (vgl. die Spielreihen der Verlage Cornelsen und Heureka/Klett und eine entsprechende Analyse in Bopp 2006). Das Spiel Global Conflicts: Palestine (Serious Games Interactive, 2007) hingegen bietet ein Beispiel für eine gelungen wirkende Verknüpfung von Lernund Erzählhandlung. Dort trifft der Spieler oder die Spielerin als junger Journalist oder junge Journalistin in Jerusalem ein, um für verschiedene Zeitungen über den Konflikt zwischen Israelis und Palästinensern zu berichten. Eine dieser Zeitungen ist pro-israelisch, eine pro-palästinensisch und eine europäisch-neutral eingestellt. Seine Informationen (z. B. zur Lage an den Grenzübergängen) erhält der Spieler durch Interviews mit mehr oder minder parteiischen Betroffenen und Experten vor Ort. Seine Artikel stellt er dann aus Textblöcken in seinem Interview-Notizblock zusammen. Dabei muss er jeweils solche Textblöcke kombinieren, die entweder einen pro-israelischen, einen pro-palästinensischen oder einen eher neutralen Standpunkt ausdrücken und den entsprechenden Artikel dann an die hierzu passende Zeitschrift senden. Danach erhält er eine Rückmeldung, inwieweit die Zeitschrift seinen Artikel als für sie passend ansieht.

In bildungsdidaktischer Hinsicht wird hiermit das Lernziel verfolgt, politische Konflikte als Interessenkonflikte analysieren und sich in einzelne Positionen hineinversetzen zu können (vgl. Breit, 1997). Die eigentlichen Lernhandlungen, nämlich die jeweiligen Einschätzungen der Interessengebundenheit einzelner Interviewaussagen, ist dabei natürlich in die Spiel- bzw. Rollenhandlung integriert: Journalisten machen sich Notizen und gehen diese dann durch, während sie ihre Artikel verfassen.

\section{Forschungsausblick}

Der vorliegende Aufsatz verdeutlicht in explorativer Weise das Potential von Storytelling als Motivationshilfe in Computerspielen. Die Entwicklung entsprechender Konzepte kann in Zukunft durch eine Vielzahl von Forschungsprojekten unterstützt werden. Erstens sind dies qualitative und quantitative Untersuchungen zu Storytelling für spezifische Lernfelder und Lerngruppen: je nach Alter, Geschlecht, Milieu etc. muss hier von unterschiedlichen Präferenzen und Rezeptionsweisen von Geschichten ausgegangen werden. Zweitens sind weitergehende Studien zum parasozialen Design von NPCs und Avataren als pädagogische Agenten 
nötig. Ist es dem Lernen z. B. förderlich, wenn ein NPC oder Avatar ähnliche Merkmale aufweist wie der Spieler? Ein dritter Schwerpunkt kann auf den besonderen Aspekten von Storytelling im Kontext von Multiplayer-Online-Lernspielen liegen, denen für die Zukunft eine zunehmende Bedeutung im Bereich von Computerlernspielen zugesprochen wird.

\section{Literaturverzeichnis}

Anderson, Craig Alan; Buckley, Katherine E. (2006). «A theoretical model of the effects and consequences of playing video games.» Playing video games. Motives, responses, and consequences. Hrsg. v. Peter Vorderer u. Jennings Bryant: Mahwah, NJ: Lawrence Erlbaum. 363-378.

Anderson, John Robert; Funke, Joachim; Plata, Guido (2007). Kognitive Psychologie, 6. Aufl. Berlin: Spektrum Akad. Verlag.

Atkins, Barry (2005). More than a game: The computer game as fictional form. Manchester: Manchester University Press.

Bandura, Alfred (1977). "Self-efficacy: toward a unifying theory of behavioral change.» Psychological Review, 84(2), 191-215.

Bateman, Chis Mark (Hrsg.). (2007). Game writing: Narrative skills for videogames. Boston, MA: Charles River Media.

Bopp, Matthias (2005). «Immersive Didaktik: Verdeckte Lernhilfen und Framingprozesse in Computerspielen.»kommunikation@gesellschaft, 6 (2): http://www. soz.uni-frankfurt.de/K.G/B2_2005_Bopp.pdf.

Bopp, Matthias (2006). «Didactic Analysis of Digital Games and Game-Based Learning.» The future of learning: Vol. 1. Affective and emotional aspects of humancomputer interaction. Game-based and innovative learning approaches. Hrsg. v. Maja Pivec. Amsterdam: IOS Press.

Breit, Gotthard (1997). Problemorientierung. Handbuch politische Bildung. Hrsg. v. Wolfgang Sander. Bonn: Bundeszentrale für politische Bildung. 63-79.

Bruner, Jerome S. (2002). Acts of meaning. Cambridge, MA: Harvard University Press.

Carr, Diane et al. (2006). Computer games: Text, narrative, and play. Cambridge, MA: Polity.

Cognition and Technology Group at Vanderbilt. (1997). The Jasper project: Lessons in curriculum, instruction, assessment, and professional development. Mahwah, NJ: Erlbaum.

Creswell, Jeff (1997). Creating worlds, constructing meaning: The Scottish storyline method. Portsmouth, NH: Heinemann.

Damasio, Antonio R. (2000). The feeling of what happens. Body and emotion in the making of consciousness. 1. Aufl., San Diego, CA: Harcourt. 
Dempsey, J.; Lucassen, B.; Gilley, W.; Rasmussen, K. (1993). «Since Malone's theory of intrinsically motivating instruction: what's the score in the gaming literature?» Journal of Educational Technology Systems, 22(2), 173-183.

Field, Syd (2005). Screenplay: The foundations of screenwriting. New York: Delta Trade Paperbacks.

Fritz, Jürgen (Hrsg.) (1995): Warum Computerspiele faszinieren. Empirische Annäherungen an Nutzung und Wirkung von Bildschirmspielen. Weinheim: Juventa.

Gee, James Paul (2004): What video games have to teach us about learning and literacy. New York, NY: Palgrave Macmillan.

Gebel, Christa (2006): «Kompetenzförderliche Potenziale unterhaltender Computerspiele». Unterrichtswissenschaft, 34(4), 290-309.

Giles, David C. (2002). "Parasocial Interaction: A Review of the Literature and a Model for Future Research.» Media Psychology, 4(3), 279-305.

Gjedde, Lisa (2006). «Story-based e-learning as a vehicle for inclusive education.» Current Developments in Technology-Assisted Education: Vol. 2. Technological Science Education, Collaborative Learning, Knowledge Management. Hrsg. v. A. Méndez-Vilas, A. Solano Martín, J. Mesa González u. J. Mesa González. Badajoz, Spain: Formatex. 1126-1130.

Heckhausen, Jutta; Heckhausen, Heinz (2006). Motivation und Handeln. 3. Aufl. Heidelberg: Springer.

Hoffmann, Anja; Göbel, Stefen; Schneider, Oliver; lurgel, Ido (2005). «Storytellingbased edutainment applications.» E-learning and virtual science centres. Hrsg. v. L. Wee Hin Tan u. R. Subramaniam. Hershey, PA: Information Science Publication. 190-214.

Hoffner, Cynthia (1996). "Children's wishful identification and parasocial interaction with favorite television characters.» Journal of Broadcasting and Electronic Media, 40, 389-402.

Horton, Donald; Wohl, Richard (1956). «Mass communication and para-social interaction. Observations on intimacy at a distance.» Psychiatry, 19(3), 215-229.

Hug, Theo (2005). «Micro-Learning and Narration. Exploring possibilities of utilization of narrations and storytelling for the designing of «micro units» and didactical micro-learning arrangements.» Paper presented at the fourth Media in Transition conference, 6-8 May, 2005, MIT, Cambridge, MA.

luppa, Nicholas; Borst, Terry (2007). Story and Simulations for Serious Games: Tales from the Trenches. Burlington, MA: Butterworth Heinemann.

Jenkins, Henry (2003). «Transmedia Storytelling: Moving characters from books to films to video games can make them stronger and more compelling.» Technology. MIT Review. http://www.technologyreview.com/printer_friendly_article. aspx?id=13052 (06.07.2007). 
Kerres, Michael (2005): "Gestaltungsorientierte Mediendidaktik und ihr Verhältnis zur Allgemeinen Didaktik.» Allgemeine Didaktik im Wandel. Hrsg. v. Peter Stadtfeld u. Bernhard Dieckmann. Bad Heilbrunn: Klinkhardt. 214-234.

Klimmt, Christoph; Vorderer, Peter (2002). «Lara ist mein Medium. Parasoziale Interaktionen mit Lara Croft im Vergleich zur Lieblingsfigur aus Film und Fernsehen.» Empirische Perspektiven der Rezeptionsforschung. Hrsg. v. Rössler, P.; Kubisch, S.; Gehrau, V.. München: Reinhard Fischer. 177-192.

Klippert, Heinz (2008). Planspiele. 10 Spielvorlagen zum sozialen, politischen und methodischen Lernen in Gruppen. Weinheim: Beltz.

Lamb, Nany (2001). The writers guide to crafting stories for children. Cincinnati, $\mathrm{OH}$ : Writer's Digest Books.

Lazzaro, Nancy (2004). «Why We Play Games: Four Keys to More Emotion Without Story.» http://www.xeodesign.com (01.03.2007).

Lee, Kwan Min; Peng, Wei (2006). «What do we know about social and psychological effects of computer games? A comprehensive review of the current literature.» Playing video games. Motives, responses, and consequences. Hrsg. v. Peter Vorderer $\mathrm{u}$. Jennings Bryant. Mahwah, NJ: Lawrence Erlbaum Associates. 325-345.

Malone, Thomas W.; Lepper, Mark R. (1987). «Making learning fun: A taxonomy of intrinsic motivations for learning.» Aptitude, Learning, and Instruction: Cognitive and Affective Process Analyses. Hrsg. v. R. Snow u. M. Farr. Hillsdale, NJ: Lawrence Erlbaum. 255-286.

McKee, Robert (1997). Story: Substance, structure, style and the principles of screenwriting. New York, N.Y.: Regan Books.

Medienpädagogischer Forschungsverband Südwest. (2005). «JIM-Studie 2005: Jugend, Information, (Multi-)Media.» http://www.mpfs.de/fileadmin/Studien/ JIM2005.pdf (04.07.2007).

Nass, Clifford; Steuer, Jonathan (1993). «Voices boxes and sources of messages: Computers and social actors.» Human Communication Research, 19(4), 504527.

Oerter, Rolf; Montada, Leo (2008). Entwicklungspsychologie. 6., vollst. überarb. Aufl. Weinheim: Beltz PVU.

Rheinberg, Falko (2005). «Intrinsische Motivation und Flow-Erleben.» Motivation und Handeln. Hrsg. v. Jutta Heckhausen u. Heinz Heckhausen. Heidelberg: Springer. 331-352.

Rojany-Buccieri, Lisa; Economy, Peter (2005). Writing children's books for dummies. Hoboken, NJ: Wiley.

Terhart, Ewald (2005). Lehr-Lern-Methoden. Eine Einführung in Probleme der methodischen Organisation von Lehren und Lernen. 4., ergänzte Auflage. Weinheim und München: Juventa. 
Vorderer, Peter; Bryant, Jennings (Hrsg.) (2006). Playing video games. Motives, responses, and consequences. Mahwah, NJ: Lawrence Erlbaum.

Tulodziecki, Gerhard; Herzig, Bardo; Blömeke, Sigrid (2004). Gestaltung von Unterricht. Eine Einführung in die Didaktik. Bad Heilbrunn: Klinkhardt.

Zillmann, D. (1996). "The psychology of suspense in dramatic exposition.» Suspense: Conceptualizations, Theoretical Analysis, and Empirical Explorations. Hrsg. v. Peter Vorderer, Hans Jürgen Wulff u. Mike Friedrichsen. Mahwah, NJ: Lawrence Erlbaum Associates. 199-231. 